Hautarzt $2021 \cdot 72: 720-728$

https://doi.org/10.1007/s00105-021-04806-4

Angenommen: 11. März 2021

Online publiziert: 13. April 2021

๑) Der/die Autor(en) 2021

\author{
Johannes Wohlrab ${ }^{1,2} \cdot$ L. Stadie ${ }^{2} \cdot$ R. H. H. Neubert ${ }^{2} \cdot$ K. Bosse ${ }^{2}$ \\ ${ }^{1}$ Universitätsklinik für Dermatologie und Venerologie, Martin-Luther-Universität Halle-Wittenberg, Halle \\ (Saale), Deutschland \\ ${ }^{2}$ An-Institut für angewandte Dermatopharmazie e. V., Martin-Luther-Universität Halle-Wittenberg, Halle \\ (Saale), Deutschland
}

\title{
Entwicklung eines Ivermectin- haltigen Saftes als Magistralrezeptur für Kinder zur Therapie der Skabies
}

Für die Behandlung einer Skabiesinfestation stehen verschiedene Arzneistoffe zur Verfügung. Neben topischen Therapieoptionen mit Permethrin (5\%), Benzoylbenzoat (10-25\%) oder Crotamiton (5-10\%) kann bei gewöhnlicher Skabies die orale Applikation von Ivermectin als Standard der Zweitlinientherapie gelten $[1,2]$. Bei immunsupprimierten Patienten, starken entzündlichen oder erosiven Begleitsymptomen bzw. bei milbenreichen Formen wird orales Ivermectin auch in der Erstlinientherapie eingesetzt [1]. Wegen der zunehmenden Verbreitung und dem damit gehäuften Auftreten der Skabies mehren sich Verdachtsmomente für eine unzureichende Wirksamkeit topischer Therapien, insbesondere bei der Verwendung von Permethrin. So findet sich eine zunehmende Zahl von Patienten, die trotz wiederholter topischer Behandlung und propagierter Umfeldsanierung nachweislich Skabiesrezidive entwickeln. Die Ursachen hierfür können vielfältig sein [3]. Dabei ist v. a. an eine fehlerhafte Anwendung der topischen Therapie zu denken, allerdings müssen auch Rezidive durch unerkannte Infestationsquellen im Umfeld des Patienten sowie möglicherweise Resistenzentwicklungen auf Permethrin bedacht werden [3-5]. Vor diesem Hintergrund hat sich bei Patienten mit Rezidivskabies die Mono- oder Kombinationstherapie mit oralem Ivermectin etabliert [6]. Als Derivat der Avermectine entfaltet Ivermectin seine Wirkung durch Blockade des Neuro- transmitters Gamma-Aminobuttersäure (GABA) und interagiert zudem direkt mit Glycin-, Histamin- und Nikotinacetylcholinrezeptoren [7]. Durch Bindung an glutamatgesteuerte Chloridkanäle in Nerven und Muskeln führt es zur Depolarisation und damit zu Lähmung sowie Absterben von Skabiesmilben [8]. Ivermectin ist für die Therapie der Skabies für Erwachsene sowie Kinder ab einem Körpergewicht von $15 \mathrm{~kg}$ in einer Dosierung von $200 \mu \mathrm{g} / \mathrm{kg}$ Körpergewicht zugelassen. Mittlerweile liegen aber auch umfangreiche Daten für die sichere und wirksame Anwendung von Ivermectin bei Kindern unter $15 \mathrm{~kg}$ Körpergewicht vor, sodass eine Anwendung bei wiederholt unwirksamer topischer Therapie als Einzelheilversuch vertretbar erscheint [9-13]. Da Ivermectin als Rezeptursubstanz nicht in Arzneibuchqualität verfügbar ist, muss auf ein Fertigarzneimittel in Form von 3 mg Tabletten zurückgegriffen werden, was eine exakte, körpergewichtsadaptierte Dosierung problematisch gestaltet. Vor diesem Hintergrund wurde ein Ivermectin-haltiger Saft für Kinder entwickelt, der aus einem Fertigarzneimittel hergestellt werden kann und hinsichtlich Stabilität, Konsistenz und Geschmack dem beabsichtigten Gebrauch angepasst wurde.

\section{Material und Methoden \\ Vehikelkonzeption}

Zur Erarbeitung eines galenischen Konzeptes wurden die Inhaltstoffe des Fertigpräparates Scabioral ${ }^{\circledR}$ (InfectoPharm Arzneimittel und Consilium $\mathrm{GmbH}$, Heppenheim, Deutschland) bzw. alternativ des aus gleicher Produktion stammenden Generikums Driponin ${ }^{\circledR}$ (Pädia GmbH, Heppenheim, Deutschland) analysiert. Durch theoretische Erwägungen, die sich aus den physikochemischen Eigenschaften des Wirkstoffs bzw. der im Fertigpräparat enthaltenen Hilfsstoffe (mikrokristalline Zellulose, vorverkleisterte Maisstärke, Butylhydroxyanisol und Magnesiumstearat) sowie den Besonderheiten der Pharmakokinetik im Kindesalter ergeben, wurde für die Entwicklung eines Saftes eine Öl-in-Wasser(O/W)-Emulsion favorisiert. Die Auswahl notwendiger zusätzlicher Hilfsstoffe erfolgt v. a. auf der Basis regulatorischer (z.B. Sicherheit, Zulassung, Verfügbarkeit, Qualität, Eignung, Preis) sowie pharmazeutischer Erfordernisse (z. B. Stabilität, Viskosität, Geschmack). Für die Herstellung einer stabilen Emulsion wurden als Emulgator Polysorbat 60 und zur Einstellung der Viskosität der Rezeptur als Gelbildner Carmellose-Natrium ausgewählt. Die Anpassung der Geschmackseigenschaften wurde durch Zusatz von Süß(Sorbitol 70, Saccharin-Natrium) und 
Aromastoffen (Himbeeraroma) eingestellt. Dabei wurde auf eine angenehme Süße und das Vermeiden eines bitteren sowie unangenehmen Bei- oder Nachgeschmacks geachtet. Zur pH-Einstellung kam Zitronensäure zum Einsatz. Zur mikrobiologischen Stabilisierung wurde die Rezeptur mit Natriumsorbat konserviert. Alle verwendeten Hilfsstoffe sind als Rezeptursubstanzen für Apotheker in Arzneibuchqualität verfügbar (WEPA Apothekenbedarf GmbH \& Co KG, Hillscheid, Deutschland bzw. Caesar \& Loretz GmbH, Hilden, Deutschland).

\section{Löslichkeitsverhalten von Ivermectin}

Ivermectin besitzt einen $\log \mathrm{P}$-Wert von 4,1 und ist somit gut fett-, aber nicht wasserlöslich. Durch eine systemische Bioverfügbarkeit von 50-60\% ergibt sich für diesen Stoff gemäß Biopharmaceutical Classification System (BCS) deshalb die Einordnung in die BCS-Klasse IV $[14,15]$. Durch die Anwendung von Ivermectin in gelöster Form lässt sich dessen systemische Bioverfügbarkeit deutlich erhöhen [16]. Deshalb wurde das Löslichkeitsverhalten des Arzneistoffs in relevanten Ölen untersucht, um eine Verarbeitung in gelöster Form auch gewährleisten, gleichzeitig aber auch die Quantität der Ölphase der Formulierung so gestalten zu können, dass die Stabilität der Emulsion erhalten bleibt. Dazu wurde das mittlere Tablettengewicht des Fertigarzneimittels bestimmt, um die äquivalente Masse einer gemörserten Tablette entsprechend $1 \mathrm{mg}$ Ivermectin exakt zu bestimmen. Die so ermittelte Masse des Mörsepulvers wurde schrittweise in einer aufsteigenden Menge Rapsöl von $0,1-0,7 \mathrm{~g}$ in $0,1-\mathrm{g}$-Schritten dispergiert. Jeweils $20 \mathrm{mg}$ des Mörsepulver-Öl-Gemisches wurden in $1,8 \mathrm{ml}$ Methanol extrahiert, anschließend auf $10 \mu \mathrm{g} / \mathrm{ml}$ verdünnt und der IvermectinGehalt mittels HPLC(Hochleistungsflüssigkeitschromatographie) vermessen.

\section{Vehikelcharakterisierung}

Zur Charakterisierung der Rezeptur wurden Spreitbarkeit, Viskosität, $\mathrm{pH}$-Eigenschaften, Brechungsindex und Dichte des Vehikels bestimmt. Die Spreitbarkeit beruht auf den rheologischen Eigenschaften einer Matrix und ermöglicht Aussagen zur Konsistenz. Hierfür wurde in eine, mit luftblasenfreier Formulierung gefüllte Dosierplatte des Extensometers (Apotec, WEPA Apothekenbedarf GmbH \& Co. KG, Hillscheid, Deutschland) ein 4-mm-Stempel eingedrückt und nach Entfernen von überschüssigem Material mit einem Kartenblatt eine skalierte Messplatte von oben mittig horizontal platziert. Nach einer Konsolidierungszeit von 1 min wurde der Radius der Spreitungsfläche mithilfe der Messskala in 4 Richtungen rechtwinklig zum Skalenverlauf abgelesen. Aus den Mittelwerten der Ausbreitungsradien wurde die Spreitbarkeit in $\mathrm{mm}^{2}$ errechnet. Die Viskosität wurde mittels Viskosimeter (Rotavisc hi-vi I, IKA Labortechnik, Staufen, Deutschland) bei konstanter Temperatur $\left(20^{\circ} \mathrm{C}\right.$ Raumtemperatur $)$ und steigender Drehzahl (0-200 rpm) vermessen. Dabei wurde die Drehzahl jeweils für 1 min gehalten, und die gemessenen Viskositäten wurden gemittelt. Die Mittelwerte wurden gegen die sich ergebene Schubspannung aufgetragen. Durch ein mit dem Viskosimeter verbundenes Thermostat (HRC 2 basic, IKA Labortechnik, Staufen, Deutschland) wurde zudem die Viskosität in Abhängigkeit von der Temperatur gemessen. Hierzu wurde die Rezeptur jeweils bei Raumtemperatur $\left(20\right.$ und $7^{\circ} \mathrm{C}$ ) mit einer konstanten Drehzahl von $35 \mathrm{rpm}$ in 3 verschiedenen Konzentrationen für 5 min vermessen $(n=4)$. Die Datenauswertung erfolgte mit der Software Labworldsoft ${ }^{\circledR} 6$ Pro (IKA Labortechnik, Staufen, Deutschland).

Die Validierung der $\mathrm{pH}$-Eigenschaften erfolgte mittels $\mathrm{pH}$-Meter (FiveEasy FE20, Mettler Toledo, Gießen, Deutschland), das vor den Messungen in einer Zweipunktmethode mit eingestellten Pufferlösungen bei $\mathrm{pH}$ 4,01 und $\mathrm{pH}$ 7,00 kalibriert wurde. Der Ziel-pH-Wert wurde entsprechend physikochemischen $\mathrm{Ge}$ gebenheiten, Stabilitäten der Inhaltstoffe und Verträglichkeit der Rezeptur als Oralia im Bereich zwischen pH 3 und 5 definiert. Der Brechungsindex als Stoffeigenschaft der Rezepturmatrix wurde mittels Refraktometer (RM40, Mettler
Toledo, Gießen, Deutschland) bei $25^{\circ} \mathrm{C}$ ermittelt. Zur exakten Dosierung der Rezeptur wurde die Zusammensetzung in Masseprozent $(\mathrm{m} / \mathrm{m})$ erarbeitet und die Dichte mittels Pyknometer nach GayLussac bestimmt. Dazu wurde das leere Pyknometer gewogen, anschließend luftblasenfrei mit der Rezepturmatrix gefüllt und erneut gewogen. Die Massedifferenz wurde zur Berechnung der Dichte herangezogen.

\section{Auswahl eines geeigneten Packmittels und Applikators}

Die chemische Stabilität von Ivermectin kann durch photochemische Reaktionen beeinträchtigt werden. Deshalb muss ein eingesetztes Packmittel Lichtschutz gewährleisten. Die für die Applikation niedrigvisköser Oralia übliche Dosiervorrichtung wie Dosierlöffel oder Becher ist wegen einer unzureichenden Dosiergenauigkeit für den beabsichtigten Einsatz der vorliegenden Rezeptur ungeeignet. Deshalb wurde eine Dosierspritze favorisiert. Bei der Auswahl und Erprobung von potenziellen Packmitteln wurde das an die Anwendungsgegebenheiten adaptierte Volumen der Rezeptur zugrunde gelegt. Dabei ergab sich für die Verarbeitung einer Tablette, enthaltend 3 mg Ivermectin, bei einer Zielkonzentration von $1 \mathrm{mg} / \mathrm{ml}$ ein Rezepturvolumen von $3 \mathrm{ml}$ bzw. bei einer Zielkonzentration von $400 \mu \mathrm{g} / \mathrm{ml}$ ein Rezepturvolumen von $7,5 \mathrm{ml}$. Deshalb wurde eine Flasche mit einem Volumen von $10 \mathrm{ml}$ mit passendem Spritzenaufsatz und geeigneten Spritzen ( $1 \mathrm{ml}$ bzw. $5 \mathrm{ml}$ Kolbenpipetten) als Packmittel favorisiert.

\section{Erarbeitung und Validierung einer analytischen Methode}

Zur Ermittlung der Wirkstoffstabilität wurde eine HPLC-Methode (Agilent Technologies 1290 Infinity LC, Waldbronn, Deutschland) entwickelt. Die Methodenentwicklung und -validierung erfolgten nach den Vorgaben internationaler Standards (USP36 NF31) und der USP(United States Pharmacopeia)Monographie für Ivermectin [17]. Zur Methodenentwicklung wurden 2 unterschiedliche Säulen (Nucleosil 120-3 C18 
$125 \times 2 \mathrm{~mm}$ und Nucleodur 100-3 C18 ec, $150 \times 4 \mathrm{~mm}$, Macgerey-Nagel, München, Deutschland) und verschiedene Verhältnisvariationen eines Lösungsmittelgemisches aus Methanol, Wasser und Ameisensäure ausgetestet. Die Validierung der Methode erfolgte mittels quantitativer Analyse mit externen Standards (Kalibrierstandards [Cals], Qualitätskontrollstandards [QCs]), die an 3 voneinander unabhängigen Tagen (3Tage-Validierung) vermessen wurden. Dadurch konnten die Richtigkeit („within-run-accuracy“, „between-run-accuracy“), Präzision („carry-over“, „lower limit of quantification“ (LLOQ), Selektivität) und Robustheit der Methode untersucht werden [18-22].

\section{Stabilitätsuntersuchungen}

Die Stabilität der Formulierungen wurde auf chemischer, organoleptischer und mikrobiologischer Ebene untersucht. Zur Bestimmung der Gleichförmigkeit des Gehalts wurden Untersuchungen in Anlehnung an die Testung einzeldosierter Arzneiformen nach European Pharmacopoeia (Ph. Eur.) 2.9.6 und 2.9.40 durchgeführt [23]. Den Ivermectin-haltigen Formulierungen wurden jeweils 3-mal $1 \mathrm{ml}$ entnommen und davon jeweils 10 äquivolume Aliquote hergestellt. Aus jeder Einzelprobe wurde Ivermectin mit Methanol extrahiert und der Gehalt bestimmt. Diese Prozedur wurde für 3 unabhängige Chargen jeder Herstellungsvariante (Fanta-Schale und Pistill mit 5 min Rührzeit, FantaSchale und Pistill mit Homogenisierung über Wasserbad $\left[40^{\circ} \mathrm{C}\right]$ und Kaltrühren, Homogenisierung mit Ultraturrax [IKA T18 basic, IKA Labortechnik, Staufen, Deutschland]) durchgeführt. Zur Bestimmung der Langzeitstabilität wurden die Formulierungen in 5-ml-Einmalspritzen abgefüllt und im Brutschrank bei $40^{\circ} \mathrm{C}$ für 5 Wochen gelagert. Dabei wurde der Füllstand auf der Spritze markiert. In der ersten Woche wurden täglich 3 Proben pro Formulierung entnommen und bezüglich Farbe, Geruch, pH-Wert, Auskristallisation, Brechungsindex, Gewicht, Homogenität, Spreitbarkeit, Phasentrennung und Gehalt überprüft. Anschließend erfolgte die

Hautarzt 2021 · 72:720-728 https://doi.org/10.1007/s00105-021-04806-4

(c) Der/die Autor(en) 2021

J. Wohlrab · L. Stadie · R. H. H. Neubert · K. Bosse

Entwicklung eines Ivermectin-haltigen Saftes als Magistralrezeptur für Kinder zur Therapie der Skabies

\section{Zusammenfassung}

Hintergrund. Zur Therapie der Skabies kann orales Ivermectin eingesetzt werden. Die Evidenz für einen sicheren und wirksamen Einsatz bei Kleinkindern im Einzelheilversuch ist erarbeitet worden und publiziert. Um eine körpergewichtsadaptierte Dosierung auch für Kinder zu gewährleisten, wurde ein Ivermectin-haltiger Saft als Magistralrezeptur entwickelt.

Material und Methoden. Da Ivermectin nicht als Reinsubstanz für die Rezeptur zur Verfügung steht, wurden wirkstoffhaltige Tabletten als Ausgangsmaterial für die Entwicklung benutzt. Die Formulierung wurde entsprechend pharmazeutischer, regulatorischer und gebrauchsorientierter Kriterien konzipiert. Zum Nachweis der chemischen Stabilität wurde eine HPLC(Hochleistungsflüssigkeitschromatographie)-Methode erarbeitet und validiert. Um die praktische Umsetzung zu erleichtern, wurden zudem Angaben zu geeigneten Packmitteln und zu Applikationshilfen erarbeitet, und die Rezeptur wurde taxiert.
Ergebnisse. Es konnte nachgewiesen werden, dass die finale Rezeptur stabil in der Apotheke hergestellt und über 3 Wochen gelagert werden kann. Es haben sich keine Bedenken bezüglich der Verträglichkeit des Rezeptursaftes ergeben. Die physikochemischen Eigenschaften und der Geschmack der Rezeptur ermöglichen den beabsichtigten Gebrauch als gut dosierbaren Saft für Kinder. Schlussfolgerung. Die entwickelte Rezeptur entspricht den Anforderungen der Apothekenbetriebsordnung ( $\$ 7$ ApBetrO) und ermöglicht eine exakte, körpergewichtsadaptierte Dosierung von oralem Ivermectin bei Kleinkindern. Untersuchungen zur Pharmakokinetik am Menschen bzw. klinische Studien zum Nachweis der Verträglichkeit und/oder Wirksamkeit liegen für die Rezeptur nicht vor.

Schlüsselwörter

Orale Applikation · Kindesalter · Off-labelAnwendung · Körpergewichtsadaptierte Dosierung $\cdot$ Applikationshilfen

\section{Development of an ivermectin-containing syrup as an extemporaneous preparation for treatment of scabies in children}

\section{Abstract}

Background. Oral ivermectin can be used to treat scabies. Evidence for safe and effective use in young children in individual treatment situations has been developed and published. In order to also ensure a body weight-adapted dosage for children, an ivermectin-containing syrup was developed as an extemporaneous preparation.

Materials and methods. Since ivermectin is not available as a pure substance for the formulation, tablets containing active ingredient were used as a basic material for development. The formulation was designed according to pharmaceutical, regulatory and use-oriented criteria. An HPLC (highpressure liquid chromatography) method was developed and validated to demonstrate chemical stability. In order to facilitate the practical implementation, information on suitable packaging material and application aids was also developed and the formulation was evaluated.
Results. It has been demonstrated that the final formulation produced in the pharmacy was stable and can be stored for 3 weeks. No concerns were raised regarding the tolerability of the syrup formulation. The physicochemical properties and the taste of the formulation allow the intended use as a well-dosed syrup for children. Conclusion. The developed formulation meets the requirements of the Apothekenbetriebsordnung (Pharmacy Work Rules; Section 7 ApBetrO) and enables an exact, body weight-adapted dosage of oral ivermectin in young children. Studies on human pharmacokinetics or clinical studies to demonstrate tolerability and/or efficacy are not available for the formulation.

Keywords

Oral administration - Children - Off-label use . Body-weight-adapted dosage · Delivery aids 


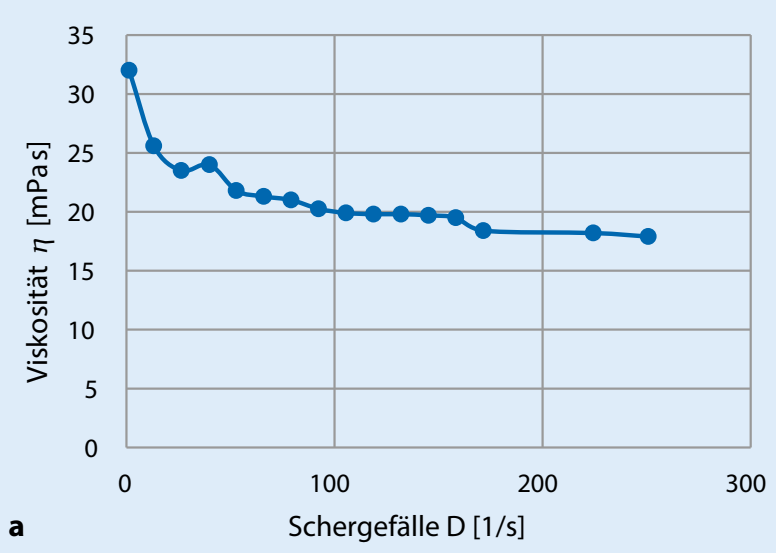

a

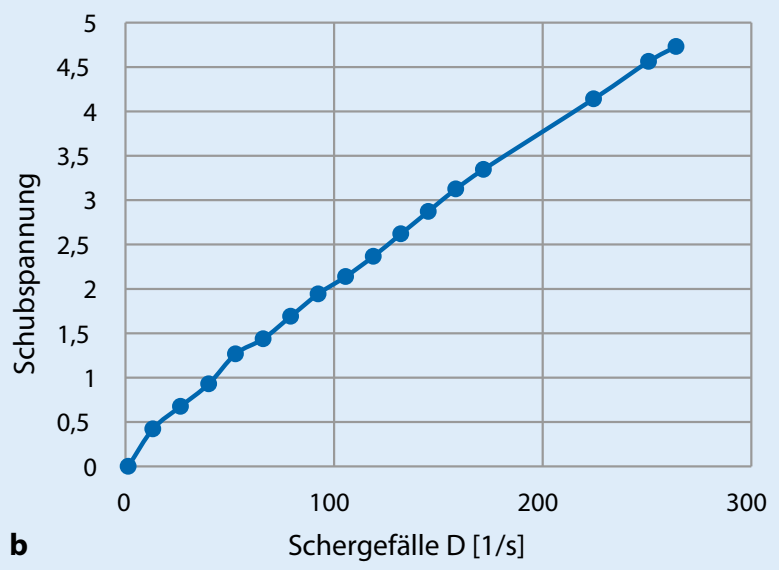

Variationen des Bezugs und damit unterschiedlicher Preise sind Abweichungen der hier berechneten Kosten möglich.

\section{Ergebnisse}

Aus praktischen Gründen wird die Ergebnispräsentation im Folgenden auf die relevanten Inhalte der letztlich ausgewählten Formulierung fokussiert und auf die Darstellungen von Daten zu weiteren 24 Vehikelvarianten verzichtet.

\section{Löslichkeitsverhalten}

Als theoretische Zielkonzentration des Ivermectin in der Ölphase wurden $11 \mu \mathrm{g} / \mathrm{ml}$ im Extraktionsmittel Methanol definiert. In $\bullet$ Tab. 1 sind die Ergebnisse der Löslichkeitsuntersuchungen wiedergegeben. Dabei zeigt sich insbesondere bei $0,2 \mathrm{~g}$ Öl eine große Abweichung vom Zielwert, die durch die ungleichmäßige Verteilung des Wirkstoffs im Mörsepulver erklärt werden kann. Dennoch zeigen die Daten, dass ab einer Ölmenge von $0,1 \mathrm{~g}$, sicher ab $0,3 \mathrm{~g}$ die Masse von $1 \mathrm{mg}$ Ivermectin gut löslich ist. Dies entspricht für 1 Tablette mit $3 \mathrm{mg}$ Ivermectin einer Mindestmenge von $0,5 \mathrm{~g}$ Öl. Gleichzeitig verdeutlichen diese Resultate, dass eine Teilung von Tabletten ein hohes Risiko einer ungenauen Dosierung beinhaltet. er (50-60 g) der Rasse „New Hampshire“ verwendet, die über 8 Tage bei $37^{\circ} \mathrm{C}$ und $55 \%$ Luftfeuchtigkeit in Paletten in einem Brutschrank bebrütet, dann eröffnet und präpariert wurden. Zur Beurteilung jeder Formulierung wurden an jeweils 6 Eiern maximal 30 min nach dem Eröffnen je $300 \mathrm{mg}$ der Testpräparation appliziert. Zur Quantifizierung der Verträglichkeit wurden mittels eines visuellen Scores die Toxizitätsparameter der CAM nach Interagency Coordinating Committee on the Validation of Alternative Methods (ICCVAM) bestimmt [27].

\section{Taxierung der Rezeptur}

Zur Taxierung der Rezeptur wurden die Apothekeneinkaufspreise für die jeweils verfügbare kleinste Abgabemenge der Bestandteile und Packmittel recherchiert und zusammen mit den Herstellungskosten entsprechend der Apothekenbetriebsordnung kalkuliert. Durch

\section{Charakterisierung}

Bezüglich der Konsistenz kann festgestellt werden, dass mit zunehmendem Emulgator-, Sorbitol- und Gelbildneranteil die Viskosität erwartungsgemäß zunimmt. Für die Herstellung der Rezeptur ist es weiterhin bedeutsam, dass die Zugabe der Ölphase sowie die Hilfsstoffe der Tablette die Viskosität erhöhen, sodass die Gelgrundlage eine sehr niedrige Viskosität hat. Zur Charakterisierung der rheologischen Eigenschaften der Rezeptur wurden ein Viskosigramm und ein Rheogramm (• Abb. 1) erstellt. Die Ergebnisse lassen auf ein pseudoplastisches Fließverhalten schließen. Dabei zeigt sich ein für Gele typisches rheologisches Verhalten einer Scherverdünnung (Thixotropie), also ein Absinken der Viskosität bei steigender Scherbeanspruchung. Dieses thixotrope Verhalten ist naler Standards zum Einsatz [27]. Es 


\begin{tabular}{llllllll}
\hline Tab. 1 & Bestimmung der Löslichkeit von Ivermectin in Rapsöl \\
\hline Rapsöl (g) & $\mathbf{0 , 1}$ & $\mathbf{0 , 2}$ & $\mathbf{0 , 3}$ & $\mathbf{0 , 4}$ & $\mathbf{0 , 5}$ & $\mathbf{0 , 6}$ & $\mathbf{0 , 7}$ \\
\hline Ivermectin & 8,818 & 4,696 & 10,241 & 9,458 & 10,443 & 10,928 & 10,928 \\
$\begin{array}{l}(\mu \mathrm{g} / \mathrm{ml}) \\
\end{array}$ & 8,811 & 4,708 & 10,236 & 9,466 & 10,452 & 10,955 & 10,955 \\
& 8,842 & 4,710 & 10,136 & 9,510 & 10,515 & 10,878 & 10,878
\end{tabular}

\section{Tab. 2 Einfluss der Temperatur auf die Viskosität}

\begin{tabular}{|c|c|c|}
\hline & \multicolumn{2}{|c|}{ Viskosität (mPa s \pm SD) } \\
\hline & $7 \pm 0,2^{\circ} \mathrm{C}$ & $20 \pm 0,3^{\circ} \mathrm{C}$ \\
\hline Placebo & $38,85 \pm 4,57$ & $23,75 \pm 2,59$ \\
\hline $400 \mu \mathrm{g} / \mathrm{ml}$ & $73,63 \pm 4,04$ & $39,78 \pm 1,77$ \\
\hline $1 \mathrm{mg} / \mathrm{ml}$ & $108,33 \pm 9,49$ & $63,58 \pm 2,75$ \\
\hline
\end{tabular}

Tab. 3 Zusammenfassung geeigneter Packmittelbestandteile

\begin{tabular}{|c|c|c|c|c|}
\hline & Genaue Bezeichnung & Anbieter & Artikel & PZN \\
\hline \multirow[t]{3}{*}{$\begin{array}{l}\text { Primärpack- } \\
\text { mittel }\end{array}$} & Glasflasche GL $1810 \mathrm{ml}$ & $\begin{array}{l}\text { WEPA, } \\
\text { Hill- } \\
\text { scheid, } \\
\text { Deutsch- } \\
\text { land }\end{array}$ & 032631 & - \\
\hline & Tropfflasche $10 \mathrm{ml}$ & $\begin{array}{l}\text { Caelo, } \\
\text { Hilden, } \\
\text { Deutsch- } \\
\text { land }\end{array}$ & 1001 & 10189381 \\
\hline & Tropfflasche $20 \mathrm{ml}$ & Caelo & 1002 & 10189398 \\
\hline $\begin{array}{l}\text { Schraubdeckel } \\
\text { mit Pipettenein- } \\
\text { satz }\end{array}$ & $\begin{array}{l}\text { + Schraubmontur weiß mit Steckeinsatz GL } 18 \\
\text { mit KISI und OV für Kolbendosierpipette }\end{array}$ & WEPA & 032403 & 01445945 \\
\hline \multirow{3}{*}{$\begin{array}{l}\text { Kolbendosier- } \\
\text { pipette }\end{array}$} & Kolbendosierpipette $1 \mathrm{ml}$ (geteilt in $0,05 \mathrm{ml}$ ) & WEPA & 032401 & 01442970 \\
\hline & Kolbendosierpipette $5 \mathrm{ml}$ (geteilt in $0,2 \mathrm{ml}$ ) & WEPA & 032402 & 01445862 \\
\hline & Dronabinol-Tropfen-Dosierspritze & Caelo & 1059 & 15257259 \\
\hline
\end{tabular}

gewünscht, da sich durch Schütteln der Zubereitung die Fließeigenschaften und damit die Dosiereigenschaften verbessern. Die Daten zeigen zudem, dass durch Abkühlung der Formulierung von 20 auf $7^{\circ} \mathrm{C}$ die Viskosität zunimmt (•Tab. 2). Somit ergibt sich bei Zimmertemperatur eine geeignet niedrige Viskosität von Fruchtsaftkonsistenz, die eine gute Dosierbarkeit gewährleistet (Vergleichswerte: Wasser ca. $1 \mathrm{mPas}$, Fruchtsaft ca. $50 \mathrm{mPa}$, Olivenöl ca. $100 \mathrm{mPa}$ ). Der $\mathrm{pH}$-Wert der Formulierung wurde auf 3,8 bis 3,9 eingestellt, sodass der Bereich von 3,0 bis 5 stabil erreicht wird. Damit sind sowohl die Stabilität der freien Glykolsäure des Carmellose-Natriums $(\mathrm{pH}>3,0)$ als auch die Wirkung von Kaliumsorbat $(\mathrm{pH}<6,0)$ und die

\section{Auswahl eines geeigneten Packmittels mit Applikator}

Durch die Analyse möglicher Packmittel wurden Primärpackmittel identifiziert, die in der Apotheke verfügbar sind, die Stabilität der Formulierung gewährleisten und die Nutzung eines Applikators zur exakten Dosierung ermöglichen (•Tab. 3).

\section{Analytische Methode}

Da Ivermectin eine sehr lipophile Substanz darstellt, wurden der in der USPMethode hohe hydrophile Anteil des Laufmittels und damit die Laufzeit reduziert. Dabei wurde auf eine klare Trennung des Wirkstoff- vom Einspritzpeak geachtet. Als Lösungsmittelgemisch wurde Ameisensäure zur Protonierung des Ivermectin eingesetzt und Acetonitril durch Methanol wegen der höheren Selektivität auf hydrophilen Säulen ausgetauscht. Zudem wurde eine Vorsäule eingesetzt, um Verunreinigungen der Analysesäule zu vermeiden. In • Tab. 4 sind die HPLC-Methoden aus USP und die erarbeitete gegenübergestellt. Durch die Optimierung der Methode und Austausch der Säule konnte zudem eine Reduktion der Laufzeit von 40 auf 5,5 min erzielt werden. Die Methodenvalidierung unter Berücksichtigung der Parameter Präzision, Richtigkeit, Selektivität, Nachweis- und Bestimmungsgrenze, „carry-over“ und Linearität wurden durch Kalibrierung ermittelt. Dabei konnte die Selektivität der Methode für Ivermectin gegenüber den Tablettierhilfsstoffen und den Gelbestandteilen dargestellt werden. Mit einem Regressionskoeffizienten von $\mathrm{R}>0,99$ in allen Untersuchungen weist die Kalibrierkurve eine gute Linearität auf. Die relativen Fehler und Variationskoeffizienten der Kalibrier- und Qualitätskontrollproben wichen um maximal 2,5\% bzw. 7,3\% vom Soll-Wert ab und erfüllten damit die Voraussetzungen für die Richtigkeit der Methode. Durch Messungen von Blindproben konnte zudem ein „carryover" ausgeschlossen werden. Die Nachweis- und Bestimmungsgrenze wurde durch das Signal-Rausch-Verhältnis der Methode ermittelt. Die Nachweisgrenze 


\begin{tabular}{|c|c|c|}
\hline & USP & Neue Methode \\
\hline Säule & $250 \times 4,6 \mathrm{~mm}(\mathrm{~L} 1 \mathrm{RP}-18)$ & $\begin{array}{l}\text { Nucleodur } 100-3 \mathrm{C} 18 \mathrm{ec}, 150 \times 4 \mathrm{~mm} \\
+ \text { Universalvorsäule EC } 3 / 4\end{array}$ \\
\hline Mobile Phase & $\begin{array}{l}\text { Acetonitril: } \mathrm{MeOH}: \mathrm{H}_{2} \mathrm{O} \\
\text { 106:55:39 (V/V/V) }\end{array}$ & $\mathrm{MeOH}: \mathrm{H}_{2} \mathrm{O}$ : Ameisensäure 93:7:0,1 (V/V/V) \\
\hline Retentionszeit & Ca. $40 \mathrm{~min}$ & $5,5 \mathrm{~min}$ \\
\hline Druck & 118 bar & 142 bar \\
\hline Flussrate & $1,5 \mathrm{ml} / \mathrm{min}$ & $0,8 \mathrm{ml} / \mathrm{min}$ \\
\hline Injektionsvolumen & $10 \mu \mathrm{l}$ & \\
\hline Detektion & UV: $245 \mathrm{~nm}$ & \\
\hline Lösungsmittel & $\mathrm{MeOH}$ & \\
\hline
\end{tabular}

Tab. 5 Berechnung der Dosierung von Ivermectin in $\mathrm{ml}$ des Saftes mit $400 \mu \mathrm{g}$ Ivermectin $/ \mathrm{ml}$ und entsprechender Rezepturmenge für 2-malige Applikation

\begin{tabular}{|lll}
$\begin{array}{l}\text { Körpergewicht } \\
(\mathbf{k g})\end{array}$ & $\begin{array}{l}\text { Zu applizierende Menge } \\
(\mathbf{m l})\end{array}$ & $\begin{array}{l}\text { Herzustellende Menge für } \mathbf{2} \text { Applikationen } \\
(\mathbf{g})\end{array}$ \\
\hline 5 & 2,5 & 7,5 \\
\hline 6 & 3 & 7,5 \\
\hline 7 & 3,5 & 7,5 \\
\hline 8 & 4 & 15 \\
9 & 4,5 & 15 \\
\hline 10 & 5 & 15 \\
11 & 5,5 & 15 \\
\hline 12 & 6 & 15 \\
\hline 13 & 6,5 & 15 \\
\hline 14 & 7 & 15 \\
\hline
\end{tabular}

kann somit mit $0,046 \mu \mathrm{g} / \mathrm{ml}$ und die Bestimmungsgrenze mit $0,220 \mu \mathrm{g} / \mathrm{ml}$ angegeben werden.

\section{Stabilitätsuntersuchungen}

Um die Gleichförmigkeit der Formulierung sicherzustellen, wurde eine Akzeptanzwertberechnung gemäß $\mathrm{Ph}$. Eur. 2.9.40 nach der Formel: $A V=|M-\bar{X}|+k s$ zugrunde gelegt [23]. Dabei ergab sich für eine Konzentration von $400 \mu \mathrm{g} / \mathrm{ml}$ ein Akzeptanzwert von 0,47 und für eine Konzentration von $1,0 \mathrm{mg} / \mathrm{ml}$ ein Akzeptanzwert von 27,82. Um die außerhalb der Akzeptanzgrenzen liegende Formulierung mit $1,0 \mathrm{mg} / \mathrm{ml}$ Ivermectin besser bewerten zu können, wurde eine Fehlersuche durchgeführt. Dazu wurde der Gehalt in einem gröBeren Probenvolumen $(0,2 \mathrm{ml})$ und anschließend wurden weitere Proben der unterschiedlichen Herstellungsmethoden vergleichend gemessen. Hier konnte keine Formulierungsvariante mit einer zeigte für die Formulierung in beiden untersuchten Konzentrationen $(400 \mu \mathrm{g} / \mathrm{ml}$ und $1,0 \mathrm{mg} / \mathrm{ml}$ ) sowohl frisch nach Herstellung als auch nach Lagerung über 5 Wochen bei $40^{\circ} \mathrm{C}$ keine Einschränkungen der mikrobiologischen Stabilität.

\section{Präklinische Untersuchungen zur Sicherheit}

Die Untersuchungen wurden gemäß Standards referenzkontrolliert durchgeführt und haben keine Hinweise oder Verdachtsmomente für ein irritatives Potenzial der Formulierung ergeben.

\section{Taxierung und Angaben zur Herstellung}

Die Herstellung erfolgt in 3 Schritten:

- Herstellung der Gelgrundlage,

- Herstellung einer öligen IvermectinLösung und

- Herstellung der Endformulierung durch Vermischen der beiden Matrices.

Für die Herstellung der Gelgrundlage sollte ein Becherglas mit Glasstab tariert und die abgewogene Menge Polysorbat 60 mit Wasser gelöst werden. Anschließend erfolgt die Zugabe der abgewogenen Mengen des bereits gelösten Kaliumsorbates und anschließend der Zitronensäure. Schließlich erfolgt die Zugabe der restlichen Inhaltstoffe unter Rühren bis ein klares, homogenes Gel entsteht. Zur Herstellung der öligen Wirkstofflösung wird eine Tablette $3 \mathrm{mg}$ Ivermectin in einer Fanta-Schale vorgelegt und mit 0,5 ml Rapsöl ergänzt. Anschließend wird die Tablette mit dem Pistill zerdrückt und im Öl angerieben. Die Endformulierung wird nun durch Zugabe der abgewogenen Menge Gelgrundlage in die Fanta-Schale mit der öligen Wirkstofflösung und der Homogenisierung der Phasen mit dem Pistill hergestellt und schließlich in das Primärpackmittel umgefüllt und verschlossen. Als Abgabepreis der Rezeptur wurden ca. 120,00€ taxiert. Der Betrag ist als Orientierung zu verstehen, und der reale Preis kann in geringem Umfang abweichen. Die Taxierung für die Ordination von „Ivermectin-Saft $400 \mu \mathrm{g} / \mathrm{ml}$ für Kinder“ ergibt sich aus den 


\begin{tabular}{|c|c|c|c|c|c|}
\hline & Anteil & Anteil in $\mathrm{g}$ & $\begin{array}{c}\text { Preis in } \\
\text { EUR }\end{array}$ & Anteil in $\mathrm{g}$ & $\begin{array}{c}\text { Preis in } \\
\text { EUR }\end{array}$ \\
\hline & & \multicolumn{2}{|c|}{$7,5 \mathrm{~g}$} & \multicolumn{2}{|c|}{$15 \mathrm{~g}$} \\
\hline Ivermectin Tablette $3 \mathrm{mg}$ & & 1 Tbl. & 79,50 & 2 Tbl. & 79,50 \\
\hline Rapsöl & & 0,5 & 0,05 & 1,00 & 0,09 \\
\hline Carmellose-Na (600 mPa s) & $0,50 \%$ & 0,075 & 0,04 & 0,075 & 0,07 \\
\hline Polysorbat 60 & $1,50 \%$ & 0,15 & 0,02 & 0,30 & 0,05 \\
\hline Sorbitol 70 & $20,00 \%$ & 1,5 & 0,22 & 3,0 & 0,43 \\
\hline Himbeer-Aroma & $0,50 \%$ & 0,0375 & 0,06 & 0,75 & 0,11 \\
\hline Saccharin-Na & $0,10 \%$ & 0,0075 & 0,01 & 0,015 & 0,01 \\
\hline Zitronensäure & $0,01 \%$ & 0,00075 & 0,01 & 0,0015 & 0,01 \\
\hline Kaliumsorbat & $0,20 \%$ & 0,015 & 0,01 & 0,03 & 0,02 \\
\hline Aqua purificata & $76,69 \%$ & 5,75175 & 0,80 & 11,5035 & 0,80 \\
\hline \multicolumn{6}{|c|}{\begin{tabular}{|l|} 
MDS: Vor Gebrauch Schütteln! 3 Wochen haltbar! \\
Dosierung: $200 \mu \mathrm{g} / \mathrm{kg}$ Körpergewicht Ivermectin am Tag 1 und Tag 10 \\
\end{tabular}} \\
\hline \multicolumn{3}{|l|}{ Glasflasche $10 \mathrm{ml}$} & 0,57 & & \\
\hline \multicolumn{3}{|l|}{ Glasflasche $20 \mathrm{ml}$} & & & 0,61 \\
\hline \multicolumn{3}{|c|}{ Schraubmontur mit Steckeinsatz } & 0,36 & & 0,36 \\
\hline \multicolumn{3}{|l|}{ Dronabinol Dosierspritze } & 3,71 & & 3,71 \\
\hline \multicolumn{3}{|c|}{ Rezeptzuschlag nach $\S 5$ Abs. 3 AMPreisV } & 6,00 & & 6,00 \\
\hline \multicolumn{3}{|l|}{ zzgl. Fixpauschale } & 8,35 & & 8,35 \\
\hline \multicolumn{3}{|l|}{ Summe (netto) } & 99,71 & & 100,12 \\
\hline \multicolumn{3}{|l|}{ zzgl. 19\% MWst } & 18,95 & & 19,03 \\
\hline \multicolumn{3}{|l|}{ Brutto-VK } & 118,66 & & 119,15 \\
\hline
\end{tabular}

Abb. $2<$ Taxierungsangaben der Magistralrezeptur. MDS (lat. misce, da, signa) mische, gib ab, beschrifte, AMPreisV Arzneimittelpreisverordnung, VKVerkaufspreis
Angaben der $\bullet$ Abb. 2, und die berechnete Dosierung des Saftes entsprechend dem Körpergewicht sowie die notwendige Rezepturmenge für eine 2-malige Applikation ergeben sich aus - Tab. 5. Die Herstellungsvorschrift für Apotheker mit exakten Angaben zur Plausibilitätsprüfung und Herstellung der Rezeptur kann über die E-Mail-Adresse „rezeptur@iadp.eu“ angefordert werden.

\section{Diskussion}

Die Ergebnisse zeigen, dass es gelungen ist, auf der Basis des verfügbaren Fertigarzneimittels in Form von Ivermectinhaltigen Tabletten eine den regulatorischen Erfordernissen entsprechende standardisierte Rezeptur zu erarbeiten. Aus der Literatur zur Pharmakologie von Ivermectin können weitgehende Rückschlüsse auf potenzielle Risiken bei der Verwendung der Rezeptur abgeleitet werden. Diese ergeben sich v. a. aus einer möglicherweise erhöhten systemischen Bioverfügbarkeit von Ivermectin durch die Lösung des Wirkstoffs in der Rezepturmatrix [16]. Im Extremfall wäre hier eine um ca. 10-20\% höhere Bioverfügbarkeit denkbar, sodass die Applikationsdosis von $200 \mu \mathrm{g} / \mathrm{kg}$ Körpergewicht nicht überschritten werden sollte. Dies ist durch die exakte Dosiermöglichkeit mittels der ausgewählten Applikationshilfe im Gegensatz zum Fertigarzneimittel auch gewährleistet. Bei Beachtung dieser Dosierempfehlung ist eine toxikologisch relevante erhöhte Bioverfügbarkeit weitgehend auszuschließen. Aufgrund der Stabilitätsdaten ist die Rezeptur mit einer Konzentration von $400 \mu \mathrm{g} / \mathrm{ml}$ klar zu präferieren. Diese erfüllt nicht nur ausnahmslos alle Kriterien einer standardisierten Rezeptur, sondern ermöglicht auch eine exakte Dosierung und wird damit allen Erfor- dernissen der praktischen Anwendung gerecht. Dennoch muss festgehalten werden, dass für die entwickelte Rezeptur bisher keine pharmakokinetischen Daten am Menschen bzw. Daten zur Verträglichkeit und/oder Wirksamkeit vorliegen. Die Bewertung der Pharmakokinetik und Sicherheit der Rezeptur ergibt sich aus tierexperimentellen Daten sowie aus Beobachtungsstudien an Kindern unter $15 \mathrm{~kg}$ Körpergewicht, die in der Indikation Onchozerkose bzw. Skabies mit systemischem Ivermectin behandelt wurden [9-13]. Der Einsatz der Magistralrezeptur sollte deshalb mit der gebotenen Vorsicht, nur bei klarer Indikationsstellung und im Bewusstsein der potenziellen Risiken als Einzelheilversuch erfolgen. Dem verordnenden Arzt sollte bewusst sein, dass das zur Herstellung der Rezepturformulierung eingesetzte Fertigarzneimittel nicht für die Anwendung bei Kindern unter $15 \mathrm{~kg}$ 
Körpergewicht zugelassen ist und eine Aufklärungspflicht der Sorgeberechtigten über die damit verbundenen Risiken sowie über Therapiealternativen besteht. Diese Aufklärung sollte schriftlich dokumentiert und gegengezeichnet archiviert werden. Um eine sachgerechte Anwendung des Rezepturarzneimittels sicherzustellen, wird die Aushändigung einer schriftlichen Gebrauchsanweisung empfohlen.

\section{Fazit für die Praxis}

\section{- Die systemische Anwendung von Ivermectin bei Kindern mit einem Körpergewicht (KG) unter $15 \mathrm{~kg}$ entspricht einer Off-label-Therapie. \\ - Dennoch gibt es eine gute Datenlage, die eine Anwendung von Ivermec- tin in einer Dosierung von $200 \mu \mathrm{g} /$ kgKG im Rahmen eines Einzelheilver- suches bei rezidivierender Skabies rechtfertigt. \\ - Zur dosisgenauen Applikation wur- de eine Rezepturformulierung als Ivermectin-Saft $400 \mu \mathrm{g} / \mathrm{ml}$ entwickelt und qualitätsvalidiert. \\ - Die 2-malige Anwendung des Saftes im Abstand von 7 bis 10 Tagen zur Therapie der Skabies wurde mit ca. $120 €$ taxiert.}

\section{Korrespondenzadresse}

\section{Prof. Dr. Johannes Wohlrab}

Universitätsklinik für Dermatologie und Venerologie, Martin-Luther-Universität HalleWittenberg

Ernst-Grube-Str. 40, 06120 Halle (Saale), Deutschland

johannes.wohlrab@medizin.uni-halle.de

Danksagung. Die Autoren bedanken sich bei Herrn Dr. Yahya Mrestani für die Unterstützung bei der Umsetzung der HPLC-Analytik sowie bei Frau Claudia Bruhne und Frau Sylke Faßhauer für die exzellente technische Assistenz bei den HET-CAM-Versuchen.

Förderung. Die vorliegenden Untersuchungen wurden vom An-Institut für angewandte Dermatopharmazie an der Martin-Luther-Universität HalleWittenberg e. V. vollumfänglich finanziert.

Funding. Open Access funding enabled and organized by Projekt DEAL.
Einhaltung ethischer Richtlinien

Interessenkonflikt. J. Wohlrab hat für Vorträge, Beratungsleistungen und anderweitige wissenschaftliche Projekte von der Firma InfectoPharm Arzneimittel und Consilium GmbH finanzielle Zuwendungen erhalten. L. Stadie, R.H.H. Neubert und K. Bosse geben an, dass kein Interessenkonflikt besteht.

Für diesen Beitrag wurden von den Autoren keine Studien an Menschen oder Tieren durchgeführt. Für die aufgeführten Studien gelten die jeweils dort angegebenen ethischen Richtlinien.

Open Access. Dieser Artikel wird unter der Creative Commons Namensnennung 4.0 International Lizenz veröffentlicht, welche die Nutzung, Vervielfältigung, Bearbeitung, Verbreitung und Wiedergabe in jeglichem Medium und Format erlaubt, sofern Sie den/die ursprünglichen Autor(en) und die Quelle ordnungsge mäß nennen, einen Link zur Creative Commons Lizenz beifügen und angeben, ob Änderungen vorgenom men wurden.

Die in diesem Artikel enthaltenen Bilder und sonstiges Drittmaterial unterliegen ebenfalls der genannten Creative Commons Lizenz, sofern sich aus der Abbildungslegende nichts anderes ergibt. Sofern das betreffende Material nicht unter der genannten Creative Commons Lizenz steht und die betreffende Handlung nicht nach gesetzlichen Vorschriften erlaubt ist, ist für die oben aufgeführten Weiterverwendungen des Materials die Einwilligung des jeweiligen Rechteinhabers einzuholen.

Weitere Details zur Lizenz entnehmen Sie bitte der Lizenzinformation auf http://creativecommons.org/ licenses/by/4.0/deed.de.

\section{Literatur}

1. Sunderkötter $\mathrm{C}$, Feldmeier $\mathrm{H}$, Folster-Holst $\mathrm{R}$ et al (2016) S1 guidelines on the diagnosis and treatment of scabies-short version. J Dtsch Dermatol Ges 14:1155-1167

2. Rosumeck S, Nast A, Dressler C (2019) Evaluation of ivermectin vs permethrin for treating scabiessummary of a cochrane review. JAMA Dermatol 155:730-732

3. Sunderkötter C, Aebischer A, Neufeld Metal (2019) Increase of scabies in Germany and development of resistant mites? Evidence and consequences. JDtsch Dermatol Ges 17:15-23

4. Nemecek R, Stockbauer A, Lexa M, Poeppl W, Mooseder G (2020) Application errors associated with topical treatment of scabies: an observational study. JDtsch Dermatol Ges 18:554-559

5. Khalil S, Abbas O, Kibbi AG, Kurban M (2017) Scabies in the age of increasing drug resistance. PLoS Negl Trop Dis 11:e5920

6. Blasco Morente G (2017) Oral lvermectin: regaining a drug for the treatment of scabies. Actas Dermosifiliogr 108:606

7. Laing R, Gillan V,DevaneyE(2017) Ivermectin_old drug, new tricks? Trends Parasitol 33:463-472

8. Suarez G, Alvarez L, Castells D et al (2013) Relative bioavailability and comparative clinical efficacy of different ivermectin oral formulations in lambs. BMC Vet Res 9:27

9. Siewe Fodjo JN, Mubiru F, Ukaga C et al (2020) Low ivermectin use among 5- to 6-year-old children: observations from door-to-door surveys in onchocerciasis-endemic regions in Africa. Int Health 12:72-75

10. Morris-Jones R (2020) Oral ivermectin for infants and children under $15 \mathrm{~kg}$ appears to be a safe and effective treatment for scabies. Br J Dermatol 182:835-836

11. Levy M, Martin L, Bursztejn AC et al (2020) Ivermectin safety in infants and children under $15 \mathrm{~kg}$ treated for scabies: a multicentric observational study. Br J Dermatol 182:1003-1006

12. Colebunders R, Wafula ST, Hotterbeekx A, SieweFodjo JN (2020) Ivermectin use in children below $15 \mathrm{~kg}$ : potential benefits for onchocerciasis and scabies elimination programmes. Br J Dermatol 182:1064

13. Stander S, Kirschstein DJ, Kohl-Sobania M et al (2020) Effectiveness and adverse events of ivermectin treatment for scabies in 30 infant patients: report from a German single centre. J Eur Acad Dermatol Venereol 34:e736-e737

14. Lo PK, Fink DW, Williams JB, Blodinger J (1985) Pharmacokinetic studies of ivermectin: effects of formulation. Vet Res Commun 9:251-268

15. Vasconcelos T, Marques S, Sarmento B (2017) The biopharmaceutical classification system of excipients. Ther Deliv 8:65-78

16. Canga AG, Sahagun A, Diez MJ et al (2007) Bioavailability of a commercial formulation of ivermectin after subcutaneous administration to sheep. Am J Vet Res 68:101-106

17. United States Pharmacopeia. USP 36-NF 31. 2013.

18. Kaza M, Karazniewicz-Lada M, Kosicka K, Siemiatkowska A, Rudzki PJ (2019) Bioanalytical method validation: new FDA guidance vs. EMA guideline. Better or worse? J Pharm Biomed Anal 165:381-385

19. Smith G (2009) Review of the 2008 European Medicines Agency concept paper on bioanalytical method validation. Bioanalysis 1:877-881

20. Tiwari G, Tiwari R (2010) Bioanalytical method validation: an updated review. Pharm Methods 1:25-38

21. Food and Drug Administration (2018) Bioanalytical method validation guidance for industry

22. Boterman M, Doig M, Breda M et al (2012) Recommendations on the interpretation of the new European Medicines Agency Guideline on Bioanalytical Method Validation by Global CRO Council for Bioanalysis (GCC). Bioanalysis 4:651-660

23. European Pharmacopoeia (Ph. Eur.). 10th Edition, 2020.

24. EMA-Committee for Medicinal Products for Human Use (2014) Guideline on stability testing for applications for variations to a marketing authorisation. EMA/CHMP/CVMP/QWP/441071/2011Rev.2.Zugegriffen: 15. Feb. 2021

25. Luepke NP (1985) Hen's egg chorioallantoic membrane test for irritation potential. Food Chem Toxicol 23:287-291

26. Spielmann H, Kalweit S, Liebsch M et al (1993) Validation study of alternatives to the Draize eye irritation test in Germany: Cytotoxicity testing and HET-CAM test with 136 industrial chemicals. Toxicol In Vitro 7:505-510

27. Interagency Coordinating Committee on the Validation of Alternative Methods (2010) ICCVAMrecommended test method protocol: hen's egg test-chorioallantoic membrane (HET-CAM) test method. https://ntp.niehs.nih.gov/iccvam/ docs/protocols/ivocular-hetcam.pdf. Zugegriffen 15. Feb. 2021 
Hier steht eine Anzeige.

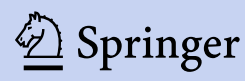

